\title{
Machen Werther und Papageno im deutschen Journalismus Schule?
}

\section{Der Stellenwert suizidfördernder und suizidpräventiver Medieneffekte in der deutschen Journalistenausbildung}

\author{
Katharina Frehmann • Markus Schäfer
}

Eingegangen: 13. Februar 2020 / Angenommen: 11. Dezember 2020 / Online publiziert: 23. Dezember 2020

(C) Der/die Autor(en) 2020

Zusammenfassung Jedes Jahr sterben in Deutschland mehr als 9000 Menschen durch Suizid. Zu den wichtigsten Präventionsmaßnahmen zählt die Weltgesundheitsorganisation eine verantwortungsvolle Medienberichterstattung über Suizide. Hintergrund hierfür sind Erkenntnisse zum „Werther-“ bzw. „Papageno-Effekt“, wonach die Medienberichterstattung über Suizide zur Entstehung oder Verhinderung weiterer Suizide beitragen kann und vor allem die Art und Weise der Berichterstattung über die Richtung der Effekte entscheidet. Aus suizidpräventiver und medienethischer Sicht scheint es daher sinnvoll, dass Medienschaffende über die möglichen Konsequenzen ihrer Berichterstattung Bescheid wissen, um diese bei ihrem Handeln berücksichtigen zu können. In der Journalistenausbildung besteht grundsätzlich ausreichend Raum für die Vermittlung solcher Inhalte. Allerdings ist zum Stellenwert suizidrelevanter Medieneffekte in der deutschen Journalistenausbildung bislang wenig bekannt. Der vorliegende Beitrag untersucht mit Hilfe einer Telefonbefragung von Verantwortlichen zentraler Institutionen der außerredaktionellen Journalistenausbildung $(n=67)$, welche Rolle die Suizidberichterstattung im Allgemeinen und die Effekte im Speziellen in Deutschland spielen. Die Ergebnisse machen deutlich, dass die Themen in vielen Bildungsangeboten bislang allenfalls unregelmäßig behandelt werden. Jedoch bestehen auf Seiten der Einrichtungen grundsätzlich Interesse und Offenheit, aber auch zusätzlicher Informationsbedarf, was in Zukunft genutzt bzw. thematisiert werden sollte.

\footnotetext{
K. Frehmann, M.A. (ه)

Institut für Sozialwissenschaften, Kommunikations- und Medienwissenschaft, Heinrich-Heine-Universität, Universitätsstraße 1, 40225 Düsseldorf, Deutschland

E-Mail: Frehmann@hhu.de

Dr. M. Schäfer

Institut für Publizistik, Johannes Gutenberg-Universität, Jakob Welder-Weg 12, 55099, 55128 Mainz,

Deutschland

E-Mail: markus.schaefer@uni-mainz.de
} 
Schlüsselwörter Journalistenausbildung · Werther-Effekt · Papageno-Effekt · Suizid · Suizidberichterstattung

\section{How established are Werther and Papageno in German journalism?}

The role of suicide-inducing and suicide-preventing media effects in German journalism training

Abstract Every year more than 9000 people in Germany die from suicide. The World Health Organization considers responsible media reporting about suicide to be one of the most important preventive measures. Research on the so-called "Werther" or "Papageno effect" shows that media coverage about suicide can contribute to the development or prevention of further suicides, suggesting that the way in which media reports about suicides can influence the direction of the effect. For example, detailed descriptions of suicides, their methods and locations tend to increase the risk of subsequent suicides. On the other hand, carefully designed articles emphasizing alternative options in suicidal crises and referring to sources of help could reduce the risk of subsequent suicides and even have a possible preventive effect. The presumed connection between media coverage and suicides is often explained by social learning theory. According to this theory, media reporting can transmit suicide models that affect the imagination and behavior of the recipients. Several scenarios are possible: For example, new behavioral and evaluation patterns (e.g., certain suicide methods or attitudes toward suicide) could be acquired by presenting previously unknown models. Further, media models can have an inhibiting or disinhibiting effect on the execution of previously acquired behavioral models. And lastly, media models could trigger behavior that has already been learned.

From the perspective of suicide prevention and media ethics, it therefore seems to be important that media professionals consider these possible effects in their daily work. But there is evidence that journalists neglect suicide prevention aspects in their reporting: In Germany, content analyses on celebrity suicides indicate that such preventive aspects are given little consideration in reporting. In addition, significant changes in suicide rates following media reports of celebrity suicides were found, that corresponded in strength and method with the previous suicide coverage. The journalistic news decision regarding suicide reports is still a black box for communication scholars. It is neither known why journalists in Germany take up the topic (selection decision), nor why they report on specific suicide cases in a certain way (construction decision). In principle, various factors can play a role in influencing the journalistic news decision. These include, for example, the characteristics of the concrete topics and events being reported on, institutional processes and constraints in the editorial offices, conventions and guidelines of the media system, the interests and positions of the recipients the reporting is directed at and the knowledge, attitudes and ideas of the journalists and editors. To be aware of the possible consequences of their reporting and to be able to take these into account when reporting about suicide, journalists need to be taught the specifics of the effects and the background about the research regarding suicide and the media.

In journalism training there is generally sufficient room to set a spotlight on such issues. However, little is known about the attention German institutions of jour- 
nalism training actually pay to suicide coverage and suicide-related media effects in particular. In Germany, the access to the profession of journalism is completely free and aspiring journalists can combine different qualification steps to become a professional, such as internships or traineeships in news rooms as well as the journalistic training at institutions like universities and journalism schools. Because of the multiverse of these possibilities and their different points of focus, contents of the training may vary. To assess whether or not suicide coverage is one of these contents, a survey of all academic journalism training institutions outside the news room (universities, colleges, academies, schools) was planned. We conducted a telephone survey of persons in charge of German institutions of journalism training. 67 of 74 persons in charge of different institutional training programs could be reached and answered questions about the role suicide coverage and suicide-related media effects actually play in German journalism training. In total, $72 \%$ of institutional training programs included suicide coverage in their teaching, but only in $25 \%$ the topic is firmly anchored in the curriculum, meaning that every cohort of students learns about it. The Werther effect is taught in $46 \%$ and the Papageno effect in only $6 \%$ of all institutions. Noticeably, programs with a broader focus on journalism in general or journalism and public relations addressed the topic more often than more specific programs concerning journalism for a special topic (e.g., sports, fashion) or special media genre (e.g., TV, radio). Respondents also gave open answers about the contexts in which suicide coverage was addressed which were later recoded into broader categories. All in all, twelve broader contexts were named by 46 respondents, which shows the heterogeneity of the different institutional training programs when it comes to this specific topic. Results show that suicide and media is mainly a topic for ethical teaching units. Further, the topic was often mentioned in combination with concrete instructions for reporting and different practical units like writing exercises. Though seldomly, the topic is also talked about in lectures and courses about media theory and effects.

There is a general interest and openness on the part of the institutions, which presented itself in various questions of the respondents during the interviews, especially about the largely unknown Papageno effect. This shows a need for additional information on behalf of the institutions which should be used and addressed in the future.

Keywords Journalism training · Werther effect · Papageno effect · Suicide · Suicide coverage

\section{Einleitung}

Suizide gehören weltweit zu den häufigsten Todesursachen. Jedes Jahr registriert die Weltgesundheitsorganisation mehr als 800.000 entsprechende Todesfälle (vgl. WHO 2018, 2019). Allein in Deutschland sterben jährlich weit mehr als 9000 Menschen durch Selbsttötungen (vgl. Statistisches Bundesamt 2019). Die Entwicklung von Maßnahmen, mit denen sich Suizide wirkungsvoll verhindern lassen, gehört daher auch hierzulande zu den größten Herausforderungen des öffentlichen Gesundheits- 
wesens. $\mathrm{Zu}$ diesen Präventionsmaßnahmen zählt die Weltgesundheitsorganisation nicht zuletzt eine verantwortungsvolle mediale Darstellung von Suiziden (vgl. WHO 2019). In den Blick der Suizidprävention rücken dabei nicht nur fiktionale Unterhaltungsformate wie etwa zuletzt die Netflix-Serie „Tote Mädchen lügen nicht“ (vgl. u. a. Bridge et al. 2019), sondern auch und insbesondere die non-fiktionale journalistische Berichterstattung über Suizide. Dass von Medienberichten grundsätzlich sowohl suizidfördernde als auch suizidpräventive Wirkungen ausgehen können, zeigen die Erkenntnisse zum „Werther-“ (vgl. Phillips 1974) bzw. „Papageno-Effekt“ (vgl. Niederkrotenthaler et al. 2010). Die Befunde deuten im Wesentlichen darauf hin, dass vor allem die Art und Weise, wie über welche Suizide berichtet wird, über die Richtung der Effekte entscheidet (vgl. Etzersdorfer et al. 2004; Niederkrotenthaler et al. 2020; Ruddigkeit 2010; Schäfer und Quiring 2015; Scherr 2013; Sisak und Värnik 2012): Während z. B. detaillierte Beschreibungen von Suizidenten, Suizidmethoden und Suizidorten die Gefahr von Folgesuiziden tendenziell erhöhen, könnten vorsichtig gestaltete Beiträge, die Handlungsalternativen betonen und auf Hilfsangebote hinweisen, die Gefahr für Folgesuizide reduzieren und möglicherweise sogar präventiv wirken.

Das Verhältnis zwischen den suizidpräventiven Interessen der Gesellschaft auf der einen und den berechtigten journalistischen Zielen einer freien und möglichst uneingeschränkten Berichterstattung auf der anderen Seite führt mitunter zu Konflikten. Für Deutschland deuten inhaltsanalytische Studien darauf hin, dass präventive Aspekte in der Berichterstattung mitunter nur wenig Berücksichtigung finden (vgl. Schäfer und Quiring 2013, 2015; Teismann et al. 2013). Welche konkreten Ursachen und Einflussfaktoren hierfür verantwortlich sind, ist bislang nicht hinreichend erforscht. Eine mögliche Erklärung könnte jedoch sein, dass die Hintergründe zum Zusammenhang von Medienberichterstattung und Suiziden und die möglichen Konsequenzen einer unvorsichtig gestalteten Suizidberichterstattung unter Medienschaffenden nicht ausreichend bekannt sind.

Nach dem journalistischen Berufsverständnis gehört insbesondere das wahrheitsgemäße Berichten über Sachverhalte oder Vorgänge zu den zentralen Aufgaben journalistischer Arbeit (vgl. Deutscher Journalisten-Verband 2020a, S. 2). Gleichzeitig ist es nur konsequent, Journalist*innen nicht gänzlich von der Verantwortung für die Inhalte, die sie produzieren, freizusprechen, sondern ihnen auch eine gewisse Fürsorgepflicht gegenüber ihren Rezipienten zuzumuten (vgl. Altmeppen et al. 2020; Ruß-Mohl 2016). Im Falle der Suizidberichterstattung bewegen sie sich somit zumindest auf den ersten Blick in einem gewissen Spannungsfeld zwischen zentralen Grundsätzen journalistischer Arbeit und den Interessen der Suizidprävention, das auch einen Konflikt zwischen gesinnungs- und verantwortungsethischem Handeln darstellen könnte (vgl. Schäfer und Potrafke 2016, S. 20). Nach Weber (1958) beinhaltet verantwortungsethisches Handeln die Übernahme von Verantwortung für die beabsichtigten und unbeabsichtigten Folgen dieses Handelns und die Ausrichtung des Verhaltens mit Blick auf diese Folgen. Gesinnungsethisches Handeln orientiert sich dagegen ausschließlich an einer übergeordneten Norm und lehnt Verantwortung für unbeabsichtigte Konsequenzen ab. Kepplinger und Knirsch (2000) fanden in ihrer Überprüfung von Webers Annahmen heraus, dass ein fehlender Konsens über die journalistischen Ziele sowie Unklarheit über die Wirkungen der Berichterstat- 
tung zu gesinnungsethischem Handeln von Journalist*innen führt. Wenn Ziele und Konsequenzen der Berichterstattung dagegen bekannt sind, handeln sie tendenziell verantwortungsethisch (vgl. Kepplinger und Knirsch 2000, S. 42).

Aus Sicht der Suizidprävention scheint es daher besonders wichtig, die vermeintlichen Widersprüche in Einklang zu bringen. Legt man die Befunde zugrunde, scheint dieses Ziel vor allem dann erreichbar, wenn Journalist*innen die suizidpräventiven Ziele ebenfalls als eine journalistische Maxime wahrnehmen und ihnen die möglichen (positiven und negativen) Konsequenzen der Suizidberichterstattung auch tatsächlich bekannt sind, um diese bei ihrem Handeln berücksichtigen zu können (vgl. Schäfer und Potrafke 2016, S. 20). Bei beidem könnte der Journalistenausbildung eine ganz entscheidende Rolle zukommen.

In der Journalistenausbildung werden nicht nur journalistische Normen vermittelt, sondern besteht prinzipiell auch ausreichend Raum für die Vermittlung von Fachkompetenzen, zu denen grundsätzlich auch wissenschaftliche Erkenntnisse zu möglichen Medienwirkungen zählen können (vgl. Meier 2018, S. 234-235). Aufgrund des freien Berufszugangs in Deutschland und der vielfältigen Bildungsangebote ist jeder journalistische Werdegang allerdings individuell. Weder gibt es ein einheitliches Berufsbild noch einen einheitlichen Ausbildungsweg (vgl. Mast 2018, S. 469). Inwieweit Journalist*innen in Deutschland also tatsächlich während ihrer Ausbildung mit dem speziellen Thema „Medien und Suizide“ in Kontakt kommen, ist eine weitgehend offene Frage. Der Beitrag geht ihr mit Hilfe einer Telefonbefragung journalistischer Bildungseinrichtungen in Deutschland ( $n=67$; Journalistenschulen, Universitäten, Hochschulen, Akademien) auf den Grund.

\section{Der Zusammenhang von Medienberichterstattung und Suiziden}

Der vermutete Zusammenhang zwischen Medienberichterstattung und Suiziden wird häufig mit der sozial-kognitiven Lerntheorie erklärt. Danach sind verschiedene Szenarien denkbar, wie sich über die Berichterstattung symbolisch vermittelte Suizidmodelle auf die Vorstellung und das Verhalten der Rezipient*innen auswirken können (vgl. Bandura 2001). So könnten neue Verhaltens- und Bewertungsmuster (z.B. bestimmte Suizidmethoden bzw. Einstellungen gegenüber dem Suizid) durch die Darstellung bislang unbekannter Modelle erworben werden. Die Darstellungen könnten hemmende oder enthemmende Wirkung bei der Umsetzung bereits erworbener Verhaltensmodelle haben und/oder im Sinne eines sozialen „Promptings“ ein bereits gelerntes und nicht unterdrücktes Verhalten auslösen (vgl. Schäfer und Quiring 2013, S. 143-144).

Je nachdem welche konkreten Suizidmodelle wie vermittelt und wie sie individuell verarbeitet werden, steigt oder sinkt das Risiko (bzw. die Chance), dass medial vermittelte Suizidmodelle zu Selbsttötungen führen (bzw. zusätzliche Suizide auf diese Weise verhindert werden). Steigt die Zahl der Suizide in der Folge von Medienberichten über Suizide an, wobei Medienwirkungen als Ursache dieser Veränderungen angenommen werden, ist von einem ,Werther-Effekt“" (vgl. Phillips 1974) die Rede; ist nach Medienberichten über Suizide ein Rückgang der Suizidzah- 
len feststellbar, der auf die Medienberichterstattung zurückführbar ist, spricht man von einem „Papageno-Effekt“ (vgl. Niederkrotenthaler et al. 2010).

Die Existenz beider Effekte gilt international als weitgehend gesichert (vgl. Niederkrotenthaler et al. 2020; Pirkis et al. 2018; Sisak und Värnik 2012) und wurde insbesondere mit Blick auf Prominentensuizide und den Printjournalismus auch immer wieder für Deutschland nachgewiesen. So fand Jonas (1992) nach Presseberichten über Prominentensuizide zwischen 1968 und 1980 einen Werther-Effekt in BadenWürttemberg. Ruddigkeit konstatierte auf Bundesebene zwischen 2001 und 2003 einen signifikanten Anstieg der Suizidzahlen nach Presseberichten der Bild-Zeitung über ,relativ prominente Personen“ (Ruddigkeit 2010, S. 267), stellte jedoch entsprechend der Annahmen der sozial-kognitiven Lerntheorie gleichzeitig auch einen Rückgang der Suizide für bestimmte Cluster von Medienbeiträgen fest, die über Suizide Unbekannter tendenziell negativ berichteten. Schäfer und Quiring (2013, 2015) fanden ebenfalls deutliche Veränderungen der Suizidzahlen nach Medienberichten über Prominentensuizide, wobei Art und Stärke der Entwicklungen mit dem Ausmaß und der Art der vorangegangenen Berichterstattung korrespondierten.

Die Wirkungseffekte beschränken sich grundsätzlich nicht nur auf Nachrichtenmedien und die non-fiktionale Berichterstattung, sondern sind auch für fiktionale Medieninhalte relevant. So stellten Schmidtke und Häfner bereits 1986 fest, dass nach Ausstrahlung einer Fernsehserie im ZDF, die den Eisenbahnsuizid eines 19-Jährigen thematisierte, die Eisenbahnsuizide von männlichen Jugendlichen in Deutschland signifikant anstiegen (vgl. Schmidtke und Häfner 1986). In den letzten Jahren wurden vor allem die amerikanische Netflix-Serie „13 Reasons Why“ und ihre möglichen Effekte diskutiert und kritisiert. Besonders der explizit dargestellte Suizid der Protagonistin und die starke Romantisierung der Tat bei gleichzeitigen Schuldzuweisungen an Mitschüler*innen und Lehrende wurden als problematisch eingestuft (vgl. Jacobson 2017, S. 8). Tatsächlich stellten verschiedene internationale Studien einen Anstieg der Suizidzahlen und des Suizidrisikos gerade für weibliche Jugendliche nach Veröffentlichung der Serie auf dem Onlineportal Netflix fest (vgl. Bridge et al. 2019; Cooper et al. 2018; Hong et al. 2018; Niederkrotenthaler et al. 2019). Die Produzenten der Serie reagierten auf die Kritik, indem sie eine allgemeine Triggerwarnung vor den Beginn der ersten Folge stellten (vgl. Bridge et al. 2019, S. 237).

Dennoch scheint das Risiko für einen Werther-Effekt nach journalistischen Medienberichten über reale Suizide größer als nach fiktionalen Mediendarstellungen, insbesondere dann, wenn es um Prominentensuizide geht (vgl. Niederkrotenthaler et al. 2020; Sisak und Värnik 2012; Stack 2002). Aus den theoretischen Annahmen und empirischen Befunden zu medialen Suiziddarstellungen lassen sich verschiedene praktische Hinweise ableiten, wie die Darstellung und Berichterstattung über Suizide aussehen sollte, um das Risiko negativer Effekte möglichst gering zu halten bzw. die Chance auf positive Wirkungen zu erhöhen. Auf dieser Grundlage hat u.a. die WHO (2017) einen Leitfaden für die mediale Berichterstattung herausgegeben, der sich gezielt an Medienschaffende wendet. Medienberichte über Suizide sollten demnach möglichst wenig prominent platziert werden und auf überdimensionierte oder reißerische Überschriften oder Teaser verzichten, um nicht unnötig Aufmerksamkeit auf negative Suizidmodelle zu lenken. Auf eine positive Darstellung oder 
Tab. 1 Kurzrichtlinien der WHO (2017) für verantwortungsvolle Berichterstattung über Suizide

\begin{tabular}{ll}
\hline Do's & Don'ts \\
\hline $\begin{array}{l}\text { Genaue Informationen zu Hilfsangeboten bereit- } \\
\text { stellen }\end{array}$ & $\begin{array}{l}\text { Suizidberichte nicht prominent platzieren und } \\
\text { nicht unangemessen } \text { oft wiederholen }\end{array}$ \\
$\begin{array}{l}\text { Publikum über Suizide und deren Prävention } \\
\text { aufklären, ohne Mythen zu verbreiten }\end{array}$ & $\begin{array}{l}\text { Keine normalisierenden oder sensationalistischen } \\
\text { Formulierungen nutzen oder Suizid als konstrukti- } \\
\text { ve Lösung für Probleme darstellen }\end{array}$ \\
$\begin{array}{l}\text { Berichte über Bewältigung von Krisen und Suizid- } \\
\text { gedanken veröffentlichen }\end{array}$ & $\begin{array}{l}\text { Genutzte Methode nicht explizit beschreiben } \\
\text { Besondere Vorsicht walten lassen, wenn über }\end{array}$ \\
$\begin{array}{l}\text { Prominentensuizide berichtet wird } \\
\text { Besondere Rücksicht } \text { auf Hinterbliebene, Famili- } \\
\text { en/Freunde des Verstorbenen nehmen }\end{array}$ & $\begin{array}{l}\text { Keine aufsehenerregenden Überschriften veröf- } \\
\text { fentlichen }\end{array}$ \\
$\begin{array}{l}\text { Beachten, dass Medienschaffende selbst von Sui- } \\
\text { zidberichten beeinflusst werden können }\end{array}$ & $\begin{array}{l}\text { Keine Fotos, Videos } \text { oder Links zu Social-Media- } \\
\text { Inhalten verwenden }\end{array}$ \\
\hline
\end{tabular}

gar Romantisierung des Suizids bzw. der Konsequenzen des Suizids sowie eine Heroisierung des Suizidenten sollten journalistische Beiträge ebenso verzichten wie auf detaillierte Informationen zu Suizidmethode, Suizidort oder Details zum Suizidenten, die Rezipientinnen und Rezipienten eine Identifikation erleichtern, also z. B. Hinweise zu Aussehen, Charakter, Lebenssituation. Der Schwerpunkt sollte stattdessen auf positiven Modellen liegen, z. B. Menschen, die suizidale Krisen durchlebt und alternative Bewältigungsstrategien gefunden haben. Informationen zu konkreten Hilfsangeboten und Anlaufstellen (z. B. Telefonnummern von Hilfe-Hotlines) sind aus suizidpräventiver Sicht ebenfalls sinnvoll, um gefährdeten Personen entsprechende Hilfestellungen zu liefern (Tab. 1).

Die Tatsache, dass solche Leitfäden zur Berichterstattung über Suizide existieren, bedeutet jedoch nicht, dass sie von Medienschaffenden auch berücksichtigt werden. Tatsächlich deuten inhaltsanalytische Studien zur medialen Darstellung von Suiziden in Deutschland darauf hin, dass die Empfehlungen zumindest mit Blick auf Prominentensuizide häufig nicht beachtet werden (vgl. Schäfer und Quiring 2013, 2015; Teismann et al. 2013). Ursache hierfür könnten verschiedene Einflussfaktoren sein, die bei der Suizidberichterstattung auf die journalistische Nachrichtenentscheidung einwirken können.

\section{Die journalistische Perspektive auf Suizide}

Die journalistische Nachrichtenentscheidung ist mit Blick auf die Suizidberichterstattung bislang weitgehend eine Black Box. Weder ist bekannt, weshalb Journalist*innen in Deutschland das Thema aufgreifen (Selektionsentscheidung), noch wissen wir, wieso sie im konkreten Fall auf eine bestimmte Art und Weise über Suizide berichten (Konstruktionsentscheidung), geschweige denn, welche konkreten Einflussfaktoren hierbei inwieweit von Bedeutung sind. Grundsätzlich können bei der journalistischen Nachrichtenentscheidung verschiedene Einflussfaktoren eine Rolle spielen. Hierzu zählen beispielsweise Eigenschaften der konkreten Themen und Ereignisse, über die berichtet wird, institutionelle Abläufe und Sachzwänge in 
den Redaktionen, Konventionen und Vorgaben des Mediensystems, Wissen, Einstellungen und Vorstellungen der Journalist*innen und Herausgeber*innen oder die Interessen und Positionen der Rezipient*innen, an die sich die Berichterstattung richtet (vgl. Schäfer 2018, S. 224-273).

In den vergangenen 30 Jahren haben diverse Autor*innen Modelle entwickelt, um (mögliche) Einflüsse auf journalistisches Handeln entlang zentraler Dimensionen zu systematisieren (vgl. z. B. Donsbach 1987, S. 112; Esser 1998, S. 27; Weischenberg 1992, S. 68). Eine Möglichkeit dabei ist, zwischen Einflussfaktoren zu unterscheiden, die auf Ebene des Individuums, der Medienorganisation, des Mediensystems und der Gesellschaft angesiedelt sind (Abb. 1).

Welche konkreten Dimensionen und Faktoren im Zuge der Suizidberichterstattung für die journalistische Selektions- und Konstruktionsentscheidung inwieweit von Belang sind, ist eine empirische Frage, die für Deutschland weitgehend unbeantwortet ist. Dies verwundert umso mehr, als fundierte Kenntnisse zur journalistischen Nachrichtenentscheidung im Zuge der Suizidberichterstattung nicht zuletzt für Akteure der Suizidprävention eine notwendige Grundlage darstellen, um zielgruppengerechte Konzepte zu entwickeln, die von Journalist*innen akzeptiert

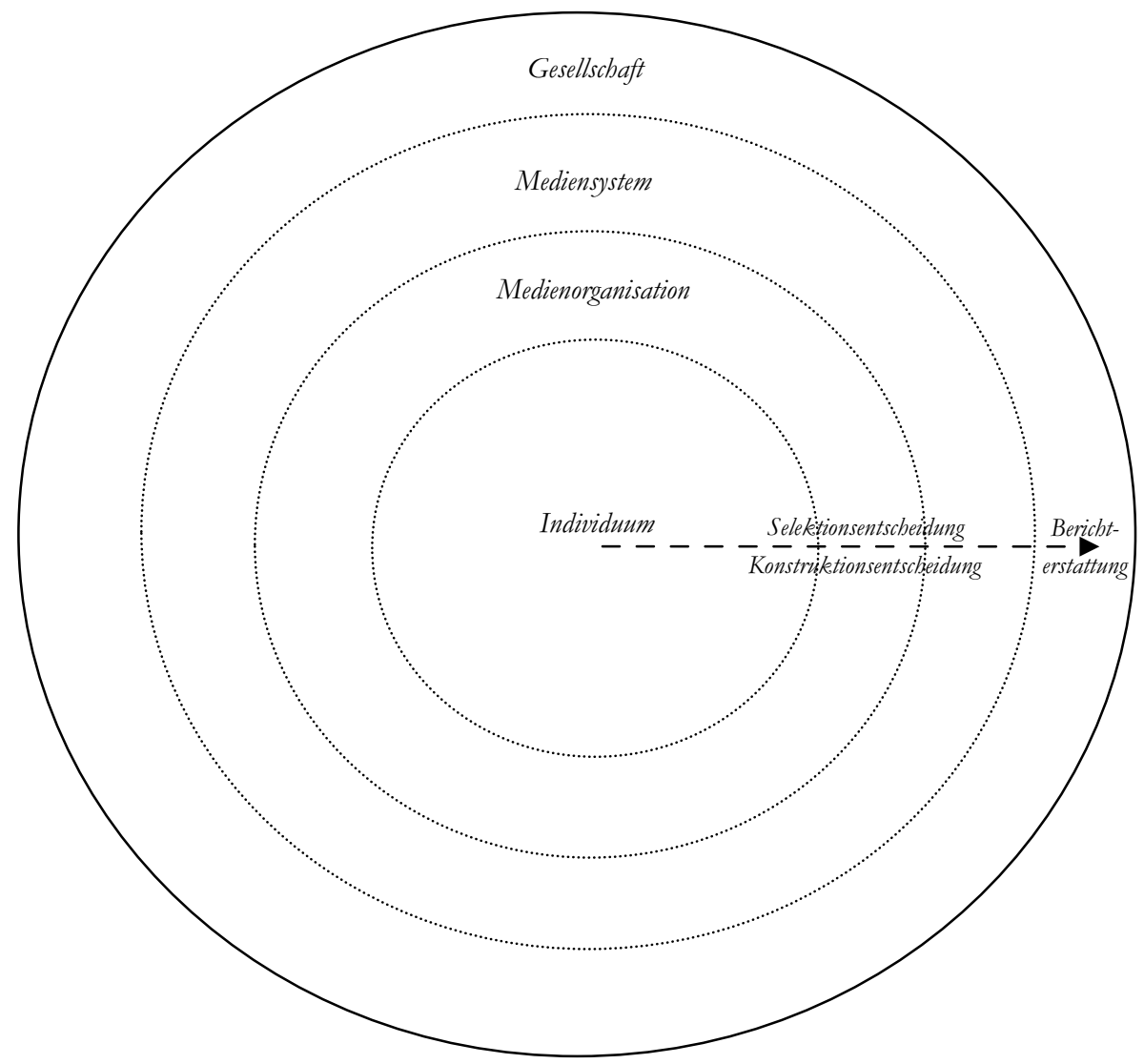

Abb. 1 Einflussebenen der journalistischen Nachrichtenentscheidung (vgl. Schäfer 2018, S. 221) 
und umgesetzt werden. Dass Letzteres keinesfalls selbstverständlich ist, zeigen die verfügbaren internationalen Studien, die sich der Frage widmen, inwieweit Journalist*innen in ihrer Berichterstattung Hinweise bestehender Leitfäden der WHO und anderer Einrichtungen einhalten. Sie kommen häufig zu dem Schluss, dass die Hinweise in der Praxis kaum bzw. nicht ausreichend Berücksichtigung finden. Sowohl Befunde zur Berichterstattung über Prominentensuizide in Deutschland (vgl. Schäfer und Quiring 2013, 2015; Teismann et al. 2013) als auch entsprechende Erkenntnisse aus anderen westlichen Ländern mit freiheitlichen Mediensystemen wie Australien (vgl. Pirkis et al. 2008) oder den USA (vgl. Tatum et al. 2010) legen nahe, dass zentrale Punkte in vielen Fällen nicht beachtet werden.

Hierfür könnte es verschiedene Ursachen auf Gesellschafts-, Mediensystem-, Medienorganisations- oder Individualebene geben. Auf Gesellschaftsebene etwa könnte das öffentliche Interesse an einer entsprechenden Berichterstattung (vermittelt über hohe Reichweiten für die berichtenden Medien) ein wichtiger Einflussfaktor sein, auf Mediensystemebene das Fehlen expliziter Hinweise in Medienkodizes oder die Spruchpraxis von Einrichtungen der Medienselbstkontrolle (vgl. Schäfer 2014; Schäfer und Potrafke 2016). Auf Ebene der Medienorganisationen könnten wirtschaftliche Überlegungen zum Publikumserfolg von Bedeutung sein, aber z.B. auch die Existenz bzw. das Fehlen einer formellen oder informellen redaktionellen Linie, Suizide (nicht) in einer bestimmten Form aufzugreifen (vgl. Altmeppen et al. 2020, S. 83-85). Auf Individualebene schließlich könnten beispielsweise das individuelle Rollenverständnis der Journalist*innen, Einstellungen zum Thema Suizid, aber auch (fehlende) Erfahrungen im Umgang mit dem Thema zu den relevanten Einflussgrößen gehören.

Denkbar wäre jedoch auch, dass die Redaktionen bzw. die Journalist*innen, die über Suizide berichten, schlichtweg nicht bzw. nicht in ausreichendem Maße über den Zusammenhang zwischen Medienberichten und Suiziden und die möglichen Folgen einer unvorsichtigen Berichterstattung informiert sind (Individualebene, Medienorganisationsebene), was nach bisherigen Erkenntnissen der Forschung ein gesinnungsethisches journalistisches Handeln tendenziell begünstigt und verantwortungsethisches Handeln erschwert (vgl. Kepplinger und Knirsch 2000, S. 42). Für diese Annahme sprechen empirische Befunde, die zeigen, dass sich die mediale Berichterstattung nach Einführung von Medienguidelines bzw. der Bereitstellung von Informationsangeboten qualitativ verbessert (vgl. Etzersdorfer und Sonneck 1998; Niederkrotenthaler und Sonneck 2007; Pirkis et al. 2009; Scherr et al. 2017), wobei dies insbesondere dann zu gelten scheint, wenn Guidelines aktiv, personalisiert und visualisiert verbreitet und zusätzlich spezielle Fortbildungen für Journalist*innen angeboten werden (vgl. Bohanna und Wang 2012, S. 195; Scherr et al. 2017). Gleichzeitig zeigen jedoch qualitative Befragungen von Journalist*innen auf internationaler Ebene, dass auf journalistischer Seite in der Praxis häufig nach wie vor Skepsis hinsichtlich der Existenz von Medieneffekten herrscht und Leitfäden unbekannt sind oder abgelehnt werden (vgl. Collings und Kemp 2010; Gandy und Terrion 2015; Jamieson et al. 2003; Subramanian 2014; Yaqub et al. 2017). Gerade hier mangelt es allerdings an weitergehenden quantitativen Studien, die repräsentative Aussagen zulassen. Ein Perspektivwechsel weg von Inhaltsanalysen, die über die Untersuchung journalistischer Produkte Rückschlüsse auf Journalist*innen zie- 
hen wollen, hin zu Befragungen, die journalistische Kenntnisse und Einstellungen direkt abfragen und deren Entstehung verfolgen, wäre jedenfalls auch mit Blick auf Deutschland ein hilfreicher Schritt.

Kenntnisse möglicher Medieneffekte ebenso wie journalistische Normen und medienethische Prinzipien sind grundsätzlich Elemente, die in der journalistischen Ausbildung vermittelt werden (können). Tatsächlich hat sich auch die Überarbeitung von Modulen in der Journalistenausbildung in anderen Ländern im universitären Kontext bereits als ein wirksames Mittel herauskristallisiert, um die suizidpräventive Qualität journalistischer Berichterstattung zu verbessern (vgl. Skehan et al. 2009, S. 198-200). Dies rückt die Rolle der Journalistenausbildung als möglichen Einflussfaktor in den Blick.

\section{Die Rolle der Journalistenausbildung}

In Deutschland, wie in den meisten freiheitlichen Demokratien, ist der Zugang zum journalistischen Beruf grundsätzlich frei. Es gibt weder eine vorgeschriebene Ausbildung noch eine formale Prüfung, die Bewerber ablegen müssten, um als Journalist*in zu arbeiten oder sich auch nur als solche(r) zu bezeichnen (vgl. Meier 2018, S. 231; Ruß-Mohl 2016). Allerdings haben sich im Laufe der Zeit bestimmte Konventionen entwickelt, die den Berufszugang regeln. Über die vergangenen Jahrzehnte ist in Deutschland eine zunehmende Professionalisierung journalistischer Arbeit feststellbar, die sich bei vielen Medienunternehmen in hohen Zugangsvoraussetzungen wie einem abgeschlossenen Studium, einem Volontariat und/oder dem Bestehen komplexer Aufnahmeprüfungen äußert. Angehende Journalist*innen wiederum können aus einer Vielzahl an Ausbildungsmöglichkeiten wählen und diese miteinander kombinieren (vgl. Meier 2018, S. 231-233; Deutscher Journalisten-Verband 2020b).

Der Großteil der deutschen Journalist*innen hat ein Studium abgeschlossen (vgl. Steindl et al. 2017, S. 414), allerdings können Schwerpunkte und Ausbildungsinhalte je nach Fachrichtung stark variieren. Einen festgelegten Lehrplan für den Journalismus gibt es zwar nicht, es herrscht aber eine relativ große Einigkeit darüber, welche Kernkompetenzen durch eine journalistische Ausbildung abgedeckt werden sollten (vgl. Dernbach und Loosen 2012, S. 13-14). Zu den zentralen Fachkompetenzen, die im Zuge der Ausbildung üblicherweise erworben werden, zählen neben dem praktischen journalistischen Handwerk (teilweise auch als Handlungskompetenz bezeichnet, siehe hierzu Nowak 2007, S. 93) nicht zuletzt auch Hintergründe zu Mediensystem und Medienrecht sowie empirische Erkenntnisse zur Wirkung der Medien. Sachkompetenzen befassen sich dagegen mit spezifischem Ressort-, Spezial- und Allgemeinwissen. Ebenfalls relevant ist die Beschäftigung mit der sozialen Verantwortung des Journalismus gegenüber Gesellschaft und Publikum aus normativer Perspektive, die sich zum Teil in der Berufsethik und Wertorientierung als eigener Vermittlungseinheit niederschlägt, teilweise aber auch den Basiskompetenzen zugeschrieben wird (vgl. Meier 2018, S. 233-236; Nowak 2007, S. 93-97). Mit dem Aufkommen neuer Herausforderungen und Arbeitsbedingungen werden in der Forschung und unter Journalist*innen selbst zudem Forderungen nach weiterer 
Kompetenzvermittlung in Technik, Management und unternehmerischen Fähigkeiten laut (vgl. Gossel 2019, S. 36-37).

Dem Aus- und Weiterbildungssystem, das Journalist*innen durchlaufen (und den Inhalten inklusive der Normen und Werte, die dort vermittelt werden), kommt beim Erwerb dieser Qualifikationen und Kompetenzen entscheidende Bedeutung zu. Zwar gibt es nach wie vor die Möglichkeit eines Quereinstiegs, als Hauptausbildungswege haben sich jedoch über Medienplattformen und Medienorganisationen hinweg vor allem der Abschluss eines Hochschulstudiums in Kombination mit dem Besuch einer Journalistenschule und/oder einem Volontariat durchgesetzt. Hinzu kommen Praktika, die Berufsanwärter heutzutage meist schon vor dem Einstieg in den Journalismus absolvieren (vgl. Donsbach 2009, S. 100-103; Lorenz 2009, S. 175). Während Qualität und Nutzen der Praktika in hohem Maße von den Gegebenheiten und Umständen abhängen, die die jeweiligen Medienorganisationen bieten, ist insbesondere bei den Studiengängen an Hoch- und Journalistenschulen, aber auch beim Volontariat - bedingt durch die Verbindung der praktischen Ausbildung in den Redaktionen mit meist organisationsübergreifenden theoretischen Ausbildungselementen, die oftmals von Berufs- und Arbeitgeberverbänden getragen und veranstaltet werden von einem deutlich höheren Standardisierungsgrad der Ausbildung auszugehen (vgl. Donsbach 2009, S. 100). Journalist*innen, die eine identische oder zumindest in weiten Teilen ähnliche Ausbildung im gleichen Ausbildungssystem durchlaufen haben, können auf ein gemeinsames Fundament an Qualifikationen zurückgreifen bzw. auf eine ähnliche Sozialisation zurückblicken, was wiederum ähnliche Berufsauffassungen und Berufsnormen begünstigt und damit für die Nachrichtenentscheidung einen nicht unerheblichen Einflussfaktor darstellt.

Was konkrete Inhalte der diversen deutschen Ausbildungsangebote betrifft, herrscht in der Wissenschaft eher Unklarheit; bisher wird wenig dazu geforscht (vgl. Gossel und Konyen 2019, S. 3). Befragungen von Journalist*innen in Ausbildung ergeben zwar ein Bild der Zufriedenheit bei gleichzeitig weitergehenden Wünschen an die Ausbildung (vgl. Neuberger und Federkeil 2011, S. 50; Gossel 2019, S. 35-38), können aber nicht objektiv wiedergeben, welche Inhalte konkret vermittelt werden. Entsprechend ist auch mit Blick auf die Rolle der Suizidprävention in der Journalistenausbildung bislang kaum etwas bekannt. Dass hier grundsätzlich ein großes Potenzial bestünde, um Medienschaffende für suizidpräventive Aspekte frühzeitig zu sensibilisieren, zeigen u.a. Erfahrungen aus Australien. Dort wurden unter Berücksichtigung der Expertisen von Akteur*innen aus Journalismus und Suizidprävention für verschiedene Journalismus-Studiengänge umfangreiche Lehrinhalte und -materialien zur Suizidberichterstattung entworfen und in die Lehre eingebunden. Eine Befragung von Journalismusstudierenden zu Gastvorträgen von Suizidexperten, die im Rahmen dieses Projekts ebenfalls stattfanden, ergab durchgehend positive Bewertungen der Vorträge. Die Befragten äußerten zudem, dass die Vorträge Einfluss auf ihre zukünftige Arbeitsweise haben werden (vgl. Skehan et al. 2009, S. 198). Auch unter den bereits ausgebildeten und arbeitenden Journalisten in Australien stellten sich besonders persönliche Schulungen und Briefings zu Suizidberichterstattungsrichtlinien als effektiv heraus, um die Aufmerksamkeit und Nutzung dieser Richtlinien in den Redaktionen zu steigern (vgl. Skehan et al. 2006, S. 31). Bohanna und Wang (2012) wiederum ermittelten in einer Metaanalyse, 
dass Medienrichtlinien am ehesten dann suizidpräventiv in die Berichterstattung eingehen, wenn sie aktiv an Journalist*innen herangetragen werden und diese Vermittlung auch (Weiter-)Bildungs-Workshops mit Journalist*innen beinhaltet - wie es im Beispiel aus Australien der Fall ist (vgl. Bohanna und Wang 2012, S. 195).

Auch im deutschsprachigen Raum gibt es bereits Hinweise, dass entsprechende Aufklärung und Schulungen von Journalisten(schüler*innen) in stärker suizidpräventiven Einstellungen und einer verbesserten Qualität der Berichterstattung münden können. Sowohl die Schulung von Journalismusstudierenden in Video- und Textform als auch ein Workshop für erfahrene Journalisten beeinflussten Arbeit, Wissen und Einstellungen der Geschulten suizidpräventiv (vgl. Scherr et al. 2017, 2019). Dies steht im Einklang mit Studien zu Bildungsinitiativen und Weiterbildungsmaßnahmen von Journalisten in diversen anderen Themenbereichen wie Wissenschaftsjournalismus, Darstellung von Muslimen und Islam oder Katastrophenberichten (vgl. Beam et al. 2015; Ewart et al. 2017; Smith et al. 2017). Inwieweit die möglichen Effekte der journalistischen Suizidberichterstattung allerdings Teil der Journalistenausbildung sind und Journalist*innen in Deutschland somit bereits im Rahmen ihrer Ausbildung auf die Herausforderungen des Spannungsfeldes vorbereitet werden, ist eine offene Frage, die es empirisch zu untersuchen gilt.

\section{Methode}

In Anbetracht der möglichen Bedeutung der journalistischen Ausbildung als Einflussfaktor der journalistischen Suizidberichterstattung geht der vorliegende Beitrag den Fragen nach, ob das Thema Suizidberichterstattung in der deutschen Journalistenausbildung aufgegriffen wird und, falls ja, in welchem Zusammenhang und mit welchen konkreten Inhalten es behandelt wird. Hierzu befragten wir Verantwortliche zentraler Institutionen der außerredaktionellen Journalistenausbildung in Deutschland telefonisch. Zur Bestimmung der Grundgesamtheit ermittelten wir zunächst alle deutschen Studiengänge und Ausbildungsinstitutionen, die sich mit Journalismus beschäftigen, indem wir eine bereits existierende Rekrutierungsliste von Scherr et al. (2017) um aktuelle Informationen ergänzten. Die Recherche ergab insgesamt 74 Bildungsangebote von 20 Universitäten, 36 Hochschulen, sechs Akademien und zwölf Journalistenschulen. Um in die Auswahl zu gelangen, mussten die Angebot explizite journalistische Lehreinheiten aufweisen und nach eigener Aussage für die journalistische Arbeit qualifizieren, wobei die konkreten inhaltlichen Schwerpunkte variieren konnten.

Die außerredaktionelle Ausbildung ist ein wichtiger Eckpfeiler der Journalistenausbildung in Deutschland. Da der Zugang zum Journalismus in Deutschland frei und hochgradig personalisierbar ist und da aktuelle repräsentative Daten für Deutschland hierzu bisweilen fehlen, lässt sich allerdings schwer quantifizieren, wie groß der Anteil der deutschen Journalist*innen tatsächlich ist, die eine Ausbildung in solchen spezifischen Studiengängen und Einrichtungen durchlaufen. Laut den verfügbaren Befragungsstudien haben in Deutschland etwa 35\% aller Journalist*innen einen Studiengang zu Journalismus oder Kommunikation absolviert, etwa $14 \%$ haben (zudem) eine Journalistenschule besucht (vgl. Hanitzsch et al. 2016, 
Tab. 2 Journalistischer Schwerpunkt der kontaktierten Bildungsangebote

\begin{tabular}{lc}
\hline & Anzahl \\
\hline Journalismus im Allgemeinen & 23 \\
Fachjournalismus (z. B. Sportjournalismus) & 20 \\
Gattungsjournalismus (z. B. TV-Journalismus) & 14 \\
Journalismus und PR & 5 \\
Medienwirtschaft/-management & 4 \\
Kommunikationswissenschaft & 1 \\
Gesamt & $\mathbf{6 7}$ \\
\hline
\end{tabular}

S. 1; Weischenberg et al. 2006, S. 353). Geht man davon aus, dass diese Zahlen über die Jahre stabil geblieben sind oder sogar dem deutschen Trend der steigenden Studienanfängerzahlen folgen (vgl. Bertelsmann Stiftung 2015, S. 9), kann also angenommen werden, dass etwa die Hälfte der deutschen Journalisten eine der abgefragten Institutionen besucht hat.

Im Zeitraum vom 10.01. bis 04.02.2019 konnten 67 Ansprechpartner*innen der ausgewählten Bildungsangebote telefonisch (bzw. in Ausnahmefällen per E-Mail) erreicht und mithilfe eines teilstandardisierten Fragebogens befragt werden (Tab. 2). Die Länge der Telefoninterviews rangierte im Mittel zwischen drei und fünf Minuten, die Befragung konnte in Extremfällen aber auch bis zu zehn Minuten lang dauern. Der Fragebogen umfasste sowohl standardisierte Fragen mit vorgegebenen Antwortmöglichkeiten als auch offene Fragen. Zunächst standen die Rolle und der Stellenwert der Suizidberichterstattung im Studium bzw. in der Ausbildung sowie die Art der vermittelten Inhalte im Zentrum. Anschließend erfragten wir spezifisch das Vorkommen der Wirkungseffekte im Curriculum (,Ist der sogenannte WertherEffekt (Papageno-Effekt) Teil des Studiums/der Ausbildung? Der Werther-Effekt (Papageno-Effekt) beschreibt das Ansteigen (Sinken) von Suizidzahlen nach Medienberichterstattung über Suizide“). Abschließend gab es Raum für ein offenes Gespräch, in dessen Verlauf auch etwaige Nachfragen der Ansprechpartner*innen zum Forschungsvorhaben und den Wirkungseffekten beantwortet wurden.

\section{Ergebnisse}

Insgesamt behandeln 48 von 67 Bildungsangeboten das Thema Suizidberichterstattung. In 17 Fällen kommt es fest im Lehrplan vor, wird also allen Jahrgängen oder Kohorten vermittelt. Dagegen greifen 31 Angebote das Thema nur unregelmäßig auf. Verteilt nach dem Schwerpunkt der Ausbildung fallen besonders die Bildungsangebote ins Auge, die den Journalismus allgemein oder in Kombination mit PR lehren: Sie thematisieren Suizide vergleichsweise häufiger als andere Bildungseinrichtungen (Tab. 3).

Hinsichtlich der expliziten Behandlung möglicher Medienwirkungseffekte konnten nicht alle Ansprechpartner*innen Auskunft geben. Oftmals begründeten sie dies damit, dass die konkrete Gestaltung von Kursinhalten den Lehrenden überlassen bleibt und nicht bis ins Detail abgesprochen wird. Es liegen 52 Antworten zum Werther- und 51 zum Papageno-Effekt vor. 24 Bildungsangebote besprechen den 
Tab. 3 Thematisierung Suizidberichterstattung nach Schwerpunkt des Bildungsangebots

\begin{tabular}{lll}
\hline & \multicolumn{2}{l}{ Suizidberichterstattung } \\
& Behandelt $(\%)$ & Nicht behandelt $(\%)$ \\
\hline Allgemeiner Journalismus $(n=23)$ & 96 & 4 \\
Gattungsspezifischer Journalismus $(n=14)$ & 57 & 43 \\
Themenspezifischer Journalismus $(n=20)$ & 55 & 45 \\
Journalismus und PR $(n=5)$ & 100 & - \\
Medienwirtschaft/-management $(n=4)$ & 50 & 50 \\
Kommunikationswissenschaft $(n=1)$ & - & 100 \\
Gesamt $(n=67)$ & $\mathbf{7 2}$ & $\mathbf{2 8}$ \\
\hline
\end{tabular}

Tab. 4 Thematisierung Werther-Effekt nach Schwerpunkt des Bildungsangebots

\begin{tabular}{lll}
\hline & Werther-Effekt & \\
& Behandelt $(\%)$ & Nicht behandelt $(\%)$ \\
\hline Allgemeiner Journalismus $(n=16)$ & 75 & 25 \\
Gattungsspezifischer Journalismus $(n=9)$ & 22 & 78 \\
Themenspezifischer Journalismus $(n=19)$ & 32 & 68 \\
Journalismus und PR $(n=3)$ & 100 & - \\
Medienwirtschaft/-management $(n=4)$ & 25 & 75 \\
Kommunikationswissenschaft $(n=1)$ & - & 100 \\
Gesamt $(n=52)$ & $\mathbf{4 6}$ & $\mathbf{5 4}$ \\
\hline
\end{tabular}

Werther-Effekt explizit, 28 tun dies nicht. Bei Betrachtung der Aufteilung nach Fachschwerpunkt wird wiederum deutlich, dass der allgemeine Journalismus und Journalismus und PR den Werther-Effekt verhältnismäßig oft thematisieren (Tab. 4). Den Papageno-Effekt greifen dagegen insgesamt nur vier Bildungsangeboten auf, 47 Angebote lehren ihn nicht.

Bei der offenen Frage, welche konkreten Inhalte die Einrichtungen zum Thema vermitteln und in welchem Zusammenhang das Thema in den Lehrplan integriert ist, konnten 46 Befragte konkrete Antworten geben. Die offenen Antworten wurden in übergeordnete Themengebiete recodiert und sind in Tab. 5 einsehbar. Unter den gegebenen Antworten fanden sich vor allem Lehrinhalte zum Thema Ethik: Die Einrichtungen sprechen das Thema Suizidberichterstattung demnach insbesondere im Rahmen von Medienethik-Seminaren und -Vorlesungen an. Dieser Punkt blieb aber oft recht vage und wurde selten weiter ausgeführt $(n=17)$. Weiterhin berichteten die Verantwortlichen häufiger davon, in der Ausbildung konkrete Handlungsanweisungen zu vermitteln, wie über das Thema Suizid berichtet werden sollte und wie nicht $(n=11)$. In diesem Zusammenhang sprachen die Ansprechpartner*innen auch viele praktische Lehreinheiten an, die über theoretisch vermitteltes Wissen hinausgehen, beispielsweise Lehrredaktionen oder Schreibübungen $(n=11)$. In neun Fällen waren zudem aktuelle oder bekannte Beispiele der Suizidberichterstattung Teil der Lehreinheiten, mehrfach wurde hier der Fall Robert Enke genannt, einmal sogar im Zusammenhang mit der Einladung seiner Witwe zu einer Vorlesung.

Die Ausbildungseinrichtungen greifen das Thema Medien und Suizide zum Teil auch in Vorlesungen und Seminaren zum Thema Medientheorie und Medienwir- 
Tab. 5 Inhaltliche Schwerpunkte bei der Thematisierung von Suizidberichterstattung in Lehrangeboten $(n=46$; Mehrfachantworten möglich)

\begin{tabular}{ll}
\hline & $\begin{array}{l}\text { Schwerpunkte } \\
\text { genannt (\%) }\end{array}$ \\
\hline Ethik & 37 \\
Konkrete Handlungsanleitung & 24 \\
Praktische Lehreinheiten & 24 \\
Nachahmer & 22 \\
Aktuelle/bekannte Beispiele der Bericht- & 20 \\
erstattung & 17 \\
Pressekodex & 17 \\
Presserecht & 17 \\
Medienwirkung/-theorie & 15 \\
Konflikt: Berichten vs. Nicht-Berichten & 11 \\
Verantwortung der Journalist*innen & 9 \\
Depression, psychische Gesundheit, Ta- & \\
buisierung & 7 \\
Verzicht auf Berichterstattung & \\
\hline
\end{tabular}

kungen auf $(n=8)$. Insgesamt zehn Gesprächspartner*innen sprachen zudem als inhaltlichen Kontext explizit die Gefahr von „Nachahmungstaten“ als möglichen Wirkungseffekt in Folge von Suizidberichterstattung an. Hierzu sei angemerkt, dass der Fragebogen erst nach der hier berichteten offenen Frage nach dem Werther- und Papageno-Effekt fragte. Diejenigen, die Nachahmer*innen erwähnten, stellten den Zusammenhang zwischen Suizidberichterstattung und Medienwirkung also selbstständig her. In sieben Fällen gingen die Ansprechpartner*innen zudem explizit auf den Konflikt zwischen Berichten und Nicht-Berichten ein: Teilweise sei es schwierig zwischen dem Bedürfnis der breiten Rezipientenmasse und dem Schutz einer kleinen Rezipientengruppe zu entscheiden, so ein Befragter. Teils gaben die Verantwortlichen auch an, dass Suizidberichterstattung nur dann zu rechtfertigen sei, wenn es sich um eine Persönlichkeit von öffentlichem Interesse handele. Interessanterweise erklärten hier drei Ansprechpartner*innen, dass in ihren Bildungsangeboten ein klarer und bedingungsloser Verzicht auf Suizidberichterstattung gelehrt werde. Fünf Einrichtungen erwähnten zudem die besondere Verantwortung der Journalist*innen bei diesem sensiblen Thema. In vier Fällen ordneten die Ansprechpartner*innen die Suizidberichterstattung übergeordneten Themen wie Depression und psychischer Gesundheit zu und sprachen die problematische Tabuisierung solcher Themengebiete an. Weiterhin nannten die Befragten Einschränkungen des Journalismus in Form des Pressekodex, mehrfach auch spezifischer in Form des Unterpunktes 8.2 (Opferschutz), aber auch Rechte der Journalist*innen beispielsweise in Form von Auskunftsansprüchen gegenüber Behörden (jeweils $n=8$ ).

Fasst man die Ergebnisse nach den theoretischen Lehreinheiten der Journalistenausbildung zusammen, vermittelt die Journalistenausbildung in Deutschland also vor allen Dingen Verantwortungs- und Fachkompetenzen zur Suizidberichterstattung: Ethische Inhalte, die Möglichkeit von Nachahmungstaten, die ethischen Richtlinien des Pressekodex, der wahrgenommene Konflikt zwischen Berichten und Nicht-Berichten und die angesprochene Verantwortung der Journalist*innen fallen alle unter 
soziale Verantwortungskompetenzen der Journalist*innen in der Gesellschaft. Zu den Fachkompetenzen zählen dagegen das konkrete Werkzeug, wie berichtet werden soll und wie nicht, praktisch angelegte Lehreinheiten und aktuelle Beispiele. Auch die theoretischen Hintergründe zu den kommunikationswissenschaftlichen Wirkungseffekten gehören zum Fachwissen über den Journalismus. Sachkompetenzen sind in diesem Fall augenscheinlich untergeordnet, nur vier Einrichtungen thematisierten Depressionen und psychische Gesundheit als eigenständige Inhalte.

\section{Fazit}

Insgesamt lässt sich festhalten, dass gut zwei Drittel der befragten Bildungsangebote auf die Suizidberichterstattung im Lehrangebot eingehen. Davon gibt aber nur die Hälfte an, dass der Inhalt fest im Curriculum verankert sei. Immerhin fast die Hälfte der antwortenden Bildungsangebote thematisiert den Werther-Effekt; der PapagenoEffekt ist dagegen weitestgehend unbekannt. Auffällig ist, dass fast alle Bildungsangebote zum allgemeinen Journalismus oder Journalismus und PR die Themen Suizidberichterstattung und Werther-Effekt mehr oder weniger regelmäßig im Lehrplan aufgreifen, themen- und gattungsspezifischer Journalismus sowie die Sonderfälle aus Medienökonomie und Kommunikationswissenschaft dagegen deutlich seltener darauf eingehen.

Inhaltlich bedeutet die Thematisierung von Suizidberichterstattung vor allen Dingen die Vermittlung von Verantwortungs- und Fachkompetenzen. Art und Kontext der vermittelten Inhalte (und ggf. Ideale) zur Suizidberichterstattung in der deutschen Journalistenausbildung sind jedoch bisweilen sehr heterogen. Von der NichtThematisierung über die Vermittlung eher theoretischer oder eher praktischer Hinweise zum journalistischen Umgang mit Suizidfällen bis hin zum Dogma eines vollständigen Verzichts auf Suizidberichterstattung ist das Spektrum recht weit. Von einer einheitlichen Handhabung und einer klaren Linie mit Blick auf die Suizidberichterstattung scheint die Journalistenausbildung in Deutschland jedenfalls noch sehr weit entfernt zu sein.

Unsere Studie beschreibt in erster Linie einen Zustand. Dass die vermittelten Ausbildungsinhalte sich auf journalistisches Handeln und damit auf journalistische Inhalte auswirken, lässt sich zwar aufgrund der dargestellten theoretischen Annahmen und früheren empirischen Erkenntnisse vermuten, wurde in unserer Studie jedoch nicht untersucht. Zum Stellenwert der Journalistenausbildung als Einflussfaktor bei journalistischen Nachrichtenentscheidungen zur Suizidberichterstattung besteht weiterhin Forschungsbedarf.

Gleiches gilt auch für den Bereich der konkret vermittelten Inhalte in der Journalistenausbildung. Die vorliegende Befragung konzentrierte sich auf Leitungsverantwortliche und Koordinator*innen der Bildungseinrichtungen, die zwar einen umfassenden Überblick über das Studienangebot haben und damit für die hier behandelte Fragestellung durchaus kompetente Ansprechpartner*innen sind. Doch verfügen sie selbst oftmals nicht über konkrete Lehrexpertise in diesem Bereich, um zusätzlich zu den allgemeinen Angaben auch konkrete Lehrinhalte einzelner Formate zu benennen und die Gründe für die Thematisierung bzw. Nichtthematisierung im spezifischen 
Kursrahmen zu erläutern. Zukünftige Untersuchungen sollten daher gezielt Lehrpersonen oder den gesamten Lehrkörper der Bildungsstätten in den Blick nehmen. Eine solche Befragung würde es ermöglichen, die Inhalte ebenso wie die Hintergründe der Lehrentscheidungen für das Aufgreifen und das Nichtaufgreifen (wie Zeitund Ressourcenknappheit, fehlende Kompetenzen oder eine zu hohe Abstraktheit des Themas) besser und detaillierter zu erfassen, als es in unserer Befragung möglich war. Dabei könnte beispielsweise auch abgefragt werden, inwieweit bestimmte Aspekte und Empfehlungen wie die der WHO (2017) in der Lehre Berücksichtigung finden. Dies könnte zu einer besseren Systematisierung und Kategorisierung der vermittelten Inhalte beitragen. Neben den hier behandelten außerredaktionellen Ausbildungsformaten sollte zudem künftig auch die Ausbildung innerhalb von Redaktionen beleuchtet werden.

Angesichts der vorhandenen Limitationen müssen unsere Befunde sicher mit Vorsicht interpretiert werden. Gleichwohl liefern sie erste Anhaltspunkte, auch was mögliche Ursachen für ein Nicht-Aufgreifen der Thematik anbelangt. Grundsätzlich gilt es zu berücksichtigen, dass die Suizidberichterstattung auch im Fall einer Thematisierung nur einen kleinen Teilbereich der journalistischen Ausbildung darstellt, in der in aller Regel in kurzer Zeit eine Vielzahl an relevanten Inhalten und Kompetenzen vermittelt werden müssen. Das dürfte den faktischen Handlungsspielraum der Institutionen wie der Lehrenden deutlich einschränken. Hinzu kommt, dass es keine auf Suizidberichte spezialisierten Fachjournalisten mit entsprechenden Sachkompetenzen gibt. Viele Journalist*innen, die über Suizide berichten, sind im konkreten Fall zum ersten Mal mit dem Thema und den damit verbundenen Entscheidungen konfrontiert. Umso wichtiger scheint es, dass zentrale Inhalte zum Umgang mit dem Thema auf breiter Basis vermittelt werden, wie es offenbar in Bildungsangeboten zum allgemeinen Journalismus im Vergleich zum Gattungs- oder Themenspezifischen Journalismus verstärkt der Fall ist.

Schließlich könnte auch das fehlende Bewusstsein für diesen nichtalltäglichen Bereich bei der konkreten Lehrplanung der praktisch-journalistischen Inhalte einen wichtigen Erklärungsansatz bieten, wie es etwa die ausführliche Aussage eines Ansprechpartners nahelegt. Dieser äußerte, dass er sich durchaus an den Werther-Effekt aus seinem theoretisch-kommunikationswissenschaftlichen Studium erinnere. Beim Lehrplan für den praktisch orientierten Journalismus habe er aber einfach nicht an diesen aus seiner Sicht doch etwas abstrakten und praxisfernen Aspekt der journalistischen Arbeit gedacht. Jetzt, wo er darüber nachdenke, halte er es aber durchaus für sinnvoll, Lehrinhalte dafür zu schaffen. Vermutlich sind gerade in den gattungsund themenspezifischen Bildungsangeboten die vermittelten Fachkompetenzen sehr stark auf die praktischen Werkzeuge des Journalismus beschränkt. Wissenschaftlich fundiertes Theoriewissen zu einem nicht direkt wahrnehmbaren Wirkungseffekt wird möglicherweise seltener vermittelt. Gerade dieses Wissen in der Journalistenausbildung braucht es aber, um Journalist*innen für die Existenz suizidpräventiver Medieneffekte zu sensibilisieren. Denn auf diese Weise könnte verhindert werden, dass Medienschaffende entsprechende Guidelines wie die der WHO als Beschränkungen in der Berichterstattung wahrnehmen und entsprechend abwehrend reagieren (vgl. Collings und Kemp 2010, S. 246; Gandy und Terrion 2015, S. 252; Subramanian 2014, S. 819-820). 
Möglicherweise existiert dieses theoretisch fundierte Wissen aber in vielen journalistischen Bildungseinrichtungen auch schlichtweg nicht in ausreichendem Maße. In der vorliegenden Studie waren viele Ansprechpartner*innen beispielsweise überrascht bei der Ansprache des Papageno-Effektes, kannten ihn nicht, interessierten sich aber teilweise für weiterführende Informationen. Einige Befragte baten nach dem Gespräch explizit um die Zusendung der Ergebnisse der vorliegenden Studie sowie von weiterem Informationsmaterial zu den Wirkungseffekten. Andere äußerten ihr Interesse an dem Thema im Verlauf des Gesprächs und dachten nach der Befragung offen über die Eingliederung des Themas in den zukünftigen Lehrplan nach. Die prinzipielle Offenheit vieler Bildungseinrichtungen, entsprechende Inhalte mit suizidpräventivem Bezug in das eigene Angebot zu integrieren, ist dabei für die Suizidprävention in Deutschland eine gute Nachricht. Denn die bislang geringe institutionelle Verankerung der Thematik vielerorts scheint folglich weniger einem Desinteresse der Verantwortlichen der Bildungsangebote geschuldet, sondern vielmehr der Tatsache, dass bislang in vielen Institutionen keine ausreichenden Informationen zu den Hintergründen der Thematik vorliegen, um ein umfassendes Lehrangebot zu den Wirkungseffekten zu gewährleisten. Zukünftige Anstrengungen der Suizidprävention in Richtung der Journalistenaus- und Fortbildung sollten also auch darauf abzielen, die Ausbildenden ausreichend zu schulen.

Förderung Die Studie wurde durch Mittel der inneruniversitären Forschungsförderung der Johannes Gutenberg-Universität Mainz (Stufe-I) unterstützt. Die Datenerhebung fand im Rahmen der Masterarbeit von Katharina Frehmann am Institut für Publizistik der Johannes Gutenberg-Universität Mainz statt.

Funding Open Access funding enabled and organized by Projekt DEAL.

Open Access Dieser Artikel wird unter der Creative Commons Namensnennung 4.0 International Lizenz veröffentlicht, welche die Nutzung, Vervielfältigung, Bearbeitung, Verbreitung und Wiedergabe in jeglichem Medium und Format erlaubt, sofern Sie den/die ursprünglichen Autor(en) und die Quelle ordnungsgemäß nennen, einen Link zur Creative Commons Lizenz beifügen und angeben, ob Änderungen vorgenommen wurden.

Die in diesem Artikel enthaltenen Bilder und sonstiges Drittmaterial unterliegen ebenfalls der genannten Creative Commons Lizenz, sofern sich aus der Abbildungslegende nichts anderes ergibt. Sofern das betreffende Material nicht unter der genannten Creative Commons Lizenz steht und die betreffende Handlung nicht nach gesetzlichen Vorschriften erlaubt ist, ist für die oben aufgeführten Weiterverwendungen des Materials die Einwilligung des jeweiligen Rechteinhabers einzuholen.

Weitere Details zur Lizenz entnehmen Sie bitte der Lizenzinformation auf http://creativecommons.org/ licenses/by/4.0/deed.de.

\section{Literatur}

Altmeppen, K. D., Brakelmann, H., \& Drössler, K. (2020). Verantwortung durch Nichtberichterstattung. Ein medienethisches Reizthema. Communicatio Socialis, 53, 80-96.

Bandura, A. (2001). Social cognitive theory of mass communication. Media Psychology, 3, 165-299.

Beam, R., Spratt, M., \& John, S. (2015). Feature reporting improves after midcareer training. Newspaper Research Journal, 36, 122-133.

Bertelsmann Stiftung (2015). Nachschulische Bildung 2030. Trends und Entwicklungsszenarien. https:// www.bertelsmann-stiftung.de/fileadmin/files/BSt/Publikationen/GrauePublikationen/LL_GP_Nach schulische_Bildung.pdf. Zugegriffen: 3. Okt. 2020. 
Bohanna, I., \& Wang, X. (2012). Media guidelines for the responsible reporting of suicide. A review of effectiveness. Crisis, 33, 190-198.

Bridge, J., Greenhouse, J., Ruch, D., Stevens, J., Ackerman, J., Sheftall, A., Horowitz, L., Kelleher, K., \& Campo, J. (2019). Association between the release of Netflix's 13 reasons why and suicide rates in the United States: an interrupted time series analysis. Journal of the American Academy of Child \& Adolescent Psychiatry, 59, 236-243.

Collings, S., \& Kemp, C. (2010). Death knocks, professional practice, and the public good: The media experience of suicide reporting in New Zealand. Social Science \& Medicine, 71, 244-248.

Cooper, T., Bard, D., Wallace, R., Gillaspy, S., \& Deleon, S. (2018). Suicide attempt admissions from a single children's hospital before and after the introduction of Netflix series 13 reasons why. Journal of Adolescent Health, 63, 688-693.

Dernbach, B., \& Loosen, W. (2012). Die didaktischen Herausforderungen in der Journalistik und der Journalistenausbildung. In B. Dernbach \& W. Loosen (Hrsg.), Didaktik der Journalistik. Konzepte, Methoden und Beispiele aus der Journalistenausbildung (S. 11-22). Wiesbaden: VS.

Deutscher Journalisten-Verband (DJV) (2020b). Journalistenschulen/Studium. https://www.djv.de/start seite/info/themen-wissen/aus-und-weiterbildung/journalistenschulenstudium.html. Zugegriffen: 10. Febr. 2020.

Deutscher Journalisten-Verband (DJV) (2020a). Berufsbild Journalistin - Journalist. Berlin: DJV.

Donsbach, W. (1987). Journalismusforschung in der Bundesrepublik: Offene Fragen trotz „Forschungsboom“. In J. Wilke (Hrsg.), Zwischenbilanz der Journalistenausbildung (S. 105-142). München: Ölschläger.

Donsbach, W. (2009). Journalist. In E. Noelle-Neumann, W. Schulz \& J. Wilke (Hrsg.), Fischer Lexikon Publizistik Massenkommunikation (S. 81-128). Frankfurt a.M.: S. Fischer.

Esser, F. (1998). Die Kräfte hinter den Schlagzeilen. Englischer und deutscher Journalismus im Vergleich. Freiburg: Karl Alber.

Etzersdorfer, E., \& Sonneck, G. (1998). Preventing suicide by influencing mass-media reporting. The Viennes experience 1980-1996. Archives of Suicide Research, 4, 67-74.

Etzersdorfer, E., Voracek, M., \& Sonneck, G. (2004). A dose-response relationship between imitational suicides and newspaper distribution. Archives of Suicide Research, 8, 137-145.

Ewart, J., O'Donnell, K., \& Chrzanowski, A. (2017). What a difference training can make: impacts of targeted training on journalists, journalism educators and journalism students' knowledge of Islam and Muslims. Journalism, 19, 762-781.

Gandy, J., \& Terrion, J.L. (2015). Journalism and suicide reporting guidelines in Canada: perspectives, partnerships and processes. International Journal of Mental Health Promotion, 17, 249-260.

Gossel, B. (2019). Teil I. Wissenschaftliche Studie: Quo Vadis Journalistenausbildung. Eine empirische Studie zur Journalistenausbildung aus Sicht junger Journalistinnen und Journalisten. In B. Gossel \& K. Konyen (Hrsg.), Quo Vadis Journalistenausbildung? Befunde und Konzepte für eine zeitgemäße Ausbildung (S. 7-68). Wiesbaden: VS.

Gossel, B., \& Konyen, K. (2019). Einleitung. In B. Gossel \& K. Konyen (Hrsg.), Quo Vadis Journalistenausbildung? Befunde und Konzepte für eine zeitgemäße Ausbildung (S. 1-6). Wiesbaden: VS.

Hanitzsch, T., Steindl, N., \& Lauerer, C. (2016). Country report: journalists in Germany. Worlds of journalism study. https://epub.ub.uni-muenchen.de/28095/1/Country\%20report\%20Germany.pdf. Zugegriffen: 3. Okt. 2020.

Hong, V., Foster, C., Magness, C., McGuire, T., Smith, P., \& King, C. (2018). 13 reasons why: viewing patterns and perceived impact among youths at risk of suicide. Psychiatric Services, 70, 107-114.

Jacobson, S. (2017). Thirteen reasons to be concerned about 13 reasons why. The Brown University Child and Adolescent Behavior Letter, 33, 8.

Jamieson, P., Jamieson, K. H., \& Romer, D. (2003). The responsible reporting of suicide in print journalism. American Behavioral Scientist, 46, 1643-1660.

Jonas, K. (1992). Modelling and suicide. A test of the Werther effect. British Journal of Social Psychology, $31,295-306$.

Kepplinger, H.M., \& Knirsch, K. (2000). Gesinnungs- und Verantwortungsethik im Journalismus. Sind Max Webers theoretische Annahmen empirisch haltbar? In M. Rath (Hrsg.), Medienethik und Medienwirkungsforschung (S. 11-44). Wiesbaden: Westdeutscher Verlag.

Lorenz, D. (2009). Journalismus. Stuttgart: Metzler.

Mast, C. (Hrsg.). (2018). ABC des Journalismus. Ein Handbuch. Köln: Herbert von Halem.

Meier, K. (2018). Journalistik. Konstanz: UVK.

Neuberger, C., \& Federkeil, G. (2011). Nach dem Bachelor: Weiterstudium oder Berufsstart? Ergebnisse der ersten bundesweiten Absolventenbefragung in der Kommunikations- und Medienwissenschaft. 
https://www.dgpuk.de/sites/default/files/Absolventenbefragung_Dokument_final_12.05-1.pdf. Zugegriffen: 21. Jan. 2020.

Niederkrotenthaler, T., \& Sonneck, G. (2007). Assessing the impact of media guidelines for reporting on suicides in Austria: interrupted time series analysis. Australian and New Zealand Journal of Psychiatry, 41, 419-428.

Niederkrotenthaler, T., Braun, M., Pirkis, J., Till, B., Stack, S., Sinyor, M., Tran, U., Voracek, M., Cheng, Q., Arendt, F., Scherr, S., Yip, P., \& Spittal, M. (2020). Association between suicide reporting in the media and suicide: systematic review and meta-analysis. BMJ, 368, m575.

Niederkrotenthaler, T., Stack, S., Till, B., Sinyor, M., Pirkis, J., Garcia, D., Rockett, I., \& Tran, U. (2019). Association of increased youth suicides in the United States with the release of 13 reasons why. JAMA Psychiatry, 76, 933-940.

Niederkrotenthaler, T., Voracek, M., Herberth, A., Till, B., Strauss, M., Etzersdorfer, E., Eisenwort, B., \& Sonneck, G. (2010). Role of media reports in completed and prevented suicide: Werther v. Papageno effects. The British Journal of Psychiatry, 197, 234-243.

Nowak, E. (2007). Qualitätsmodell für die Journalistenausbildung. Kompetenzen, Ausbildungswege, Fachdidaktik. Unveröffentlichte Dissertation, Universität Dortmund.

Phillips, D. (1974). The influence of suggestion on suicide: substantive and theoretical implications of the Werther effect. American Sociological Review, 39, 340-354.

Pirkis, J., Blood, R. W., Dare, A., \& Holland, K. (2008). The media monitoring project. Changes in media reporting of suicide and mental health and illness in Australia: 2000-2006. Canberra: Commonwealth Department of Health and Aged Care.

Pirkis, J., Blood, R. W., Sutherland, G., \& Currier, D. (2018). Suicide and the news and information media. A critical review. Canberra: Mindframe.

Pirkis, J., Dare, A., Blood, R. W., Rankin, B., Williamson, M., Burgess, P., \& Jolley, D. (2009). Changes in media reporting of suicide in Australia between 2000/01 and 2006/07. Crisis, 30, 25-33.

Ruddigkeit, A. (2010). Der umgekehrte Werther-Effekt. Eine quasi-experimentelle Untersuchung von Suizidberichterstattung und deutscher Suizidrate. Publizistik, 55, 25-273.

Ruß-Mohl, S. (2016). Journalismus. Das Lehr- und Handbuch. Frankfurt a.M.: Frankfurter Allgemeine Buch.

Schäfer, M. (2014). Persönlichkeitsschutz vor Suizidprävention: Die Spruchpraxis des Deutschen Presserats zu Beschwerden zur Suizidberichterstattung. In E. Baumann, M. Hastall, C. Rossmann \& A. Sowka (Hrsg.), Gesundheitskommunikation als Forschungsfeld der Kommunikations- und Medienwissenschaft (S. 163-175). Baden-Baden: Nomos.

Schäfer, M. (2018). Medienhype „Hirndoping“? Die Rolle der Journalisten in der gesellschaftlichen Debatte um Neuroenhancement. Baden-Baden: Nomos.

Schäfer, M., \& Potrafke, S. (2016). Welche Rolle spielt die Suizidprävention? Ein internationaler Vergleich von Pressekodizes im Hinblick auf Richtlinien zur Suizidberichterstattung. In A.-L. Camerini, R. Ludolph \& F. Rothenfluh (Hrsg.), Gesundheitskommunikation im Spannungsfeld zwischen Theorie und Praxis (S. 17-28). Baden-Baden: Nomos.

Schäfer, M., \& Quiring, O. (2013). Gibt es Hinweise auf einen „Enke-Effekt“? Die Presseberichterstattung über den Suizid von Robert Enke und die Entwicklung der Suizidzahlen in Deutschland. Publizistik, $58,141-160$.

Schäfer, M., \& Quiring, O. (2015). The press coverage of celebrity suicide and the development of suicide frequencies in Germany. Health Communication, 30, 1149-1158.

Scherr, S. (2013). Medien und Suizide: Überblick über die kommunikationswissenschaftliche Forschung zum Werther-Effekt. Suizidprophylaxe, 40, 96-107.

Scherr, S., Arendt, F., \& Schäfer, M. (2017). Supporting reporting: on the positive effects of text- and video-based awareness material on responsible journalistic suicide news writing. Archives of Suicide Research, 21, 646-658.

Scherr, S., Markiewitz, A., \& Arendt, F. (2019). Effectiveness of a workshop intervention on responsible reporting on suicide among Swiss media professionals. Crisis, 40, 446-450.

Schmidtke, A., \& Häfner, H. (1986). Die Vermittlung von Selbstmordmotivation und Selbstmordhandlungen durch fiktive Modelle. Die Folgen der Fernsehserie „Tod eines Schülers“. Nervenarzt, 57, 502-510.

Sisak, M., \& Värnik, A. (2012). Media roles in suicide prevention: a systematic review. International Journal of Environmental Research and Public Health, 9, 123-138.

Skehan, J., Burns, L.S., \& Hazell, T. (2009). The response ability project: integrating the reporting of suicide and mental illness into journalism curricula. Journalism \& Mass Communication Educator, 64, 192-204. 
Skehan, J., Greenhalgh, S., Hazell, T., \& Pirkis, J. (2006). Reach, awareness and uptake of media guidelines for reporting suicide and mental illness: an Australian perspective. International Journal of Mental Health Promotion, 8(4), 29-35.

Smith, H., Menezes, S., \& Gilbert, C. (2017). Science training and environmental journalism today: effects of science journalism training for midcareer professionals. Applied Environmental Education \& Communication, 17, 161-173.

Stack, S. (2002). Media coverage as a risk factor in suicide. Injury Prevention, 8, 957-971.

Statistisches Bundesamt (2019). Todesursachen. Suizide. https://www.destatis.de/DE/Themen/GesellschaftUmwelt/Gesundheit/Todesursachen/Tabellen/suizide.html. Zugegriffen: 16. Dez. 2019.

Steindl, N., Lauerer, C., \& Hanitzsch, T. (2017). Journalismus in Deutschland: Aktuelle Befunde zu Kontinuität und Wandel im deutschen Journalismus. Publizistik, 62, 401-423.

Subramanian, R. (2014). Covering mental illness. Challenges and solutions. Journalism Practice, 8, $809-825$.

Tatum, P., Canetto, S. S., \& Slater, M. (2010). Suicide coverage in U.S. newspapers following the publication of the media guidelines. Suicide and Life-Threatening Behavior, 40, 524-534.

Teismann, T., Schwidder, J., \& Willutzki, U. (2013). Mediale Berichterstattung über den Suizid von Robert Enke. Zeitschrift für Gesundheitspsychologie, 21, 113-121.

Weber, M. (1958). Politik als Beruf. Berlin: Duncker \& Humblot.

Weischenberg, S. (1992). Mediensysteme, Medienethik, Medieninstitutionen. Journalistik. Theorie und Praxis aktueller Medienkommunikation, Bd. 1. Opladen: Westdeutscher Verlag.

Weischenberg, S., Malik, M., \& Scholl, A. (2006). Journalismus in Deutschland 2005. Zentrale Befunde der aktuellen Repräsentativbefragung deutscher Journalisten. Media Perspektiven, 7, 346-361.

World Health Organization (WHO) (2018). Global health estimates 2016. Global deaths by cause, age, sex, by country and by region, 2000-2016. https://www.who.int/healthinfo/global_burden_disease/ GHE2016_Deaths_Global_2000_2016.xls?ua=1. Zugegriffen: 31. Mai 2019.

World Health Organization (WHO) (2019). Suicide. https://www.who.int/news-room/fact-sheets/detail/ suicide. Zugegriffen: 29. Nov. 2019.

World Health Organization (WHO) (2017). Preventing suicide: a resource for media professionals. Update 2017. Genf: WHO.

Yaqub, M. M., Beam, R., \& John, S. L. (2017). "We report the world as it is, not as we want it to be". Journalists' negotiation of professional practices and responsibilities when reporting on suicide. Journalism. https://doi.org/10.1177/1464884917731957.

Katharina Frehmann ist wissenschaftliche Mitarbeiterin am Institut für Sozialwissenschaften der Heinrich-Heine-Universität Düsseldorf.

Dr. Markus Schäfer ist wissenschaftlicher Mitarbeiter am Institut für Publizistik der Johannes GutenbergUniversität Mainz. 